\title{
Mechanical properties of nacre constituents and their impact on mechanical performance
}

\author{
François Barthelat, Chun-Ming Li, Claudia Comi, ${ }^{\text {a) }}$ and Horacio D. Espinosa ${ }^{\text {b) }}$ \\ Department of Mechanical Engineering, Northwestern University, Evanston, Illinois 60208-3111
}

(Received 18 July 2005; accepted 23 February 2006)

\begin{abstract}
The mechanical properties of nacre constituents from red abalone were investigated. Electron microscopy studies revealed that the tablets are composed of single-crystal aragonite with nanograin inclusions. Both nanoasperities and aragonite bridges are present within the interfaces between the tablets. By means of nanoindentation and axial compression tests, we identified single tablet elastic and inelastic properties. The elastic properties are very similar to those of single-crystal aragonite. However, their strength is higher than previously reported values for aragonite. A finite element model of the interface accounting for nanoasperities and the identified properties revealed that the nanoasperities are strong enough to withstand climbing and resist tablet sliding, at least over the initial stages of deformation. Furthermore, it was observed that the model over-predicts strength and under-predicts ductility. Therefore, we conclude that other interface features must be responsible for the enhanced performance of nacre over its constituents.
\end{abstract}

\section{INTRODUCTION}

Some structural materials found in nature exhibit remarkable mechanical performance. It is believed that these are the results of material design over several length scales, organized in a hierarchical fashion. Nacre is a typical example of such biomaterials. It exhibits remarkable properties not achievable in current man-made ceramic composites. For this reason, the investigation of nacre mechanics is becoming the focus of a growing number of research groups within the materials science community.

Nacre is the iridescent material found in the inner layer of some seashells such as oyster or abalone. It is a composite material mainly composed of an aragonite phase (about 95 vol\%) arranged in microscopic polygonal flat tablets, bonded together by a biopolymer thin film (Fig. 1). Aragonite is a very fragile ceramic. However the addition of a small amount of the organic polymer and a well-designed microstructure results in a material with strength and toughness 20-30 times that of monolithic aragonite. ${ }^{1}$ The toughening mechanisms involved in nacre have been the focus of significant research efforts in the past ten years. Several possible deformation mechanisms have been suggested, but definite identification of

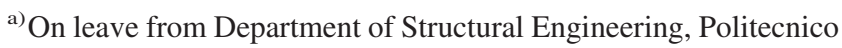
di Milano, Milano, Italy.

b) Address all correspondence to this author.

e-mail: espinosa@northwestern.edu

DOI: $10.1557 / J M R .2006 .0239$
}

the microstructural features leading to this performance is yet to be established. ${ }^{2}$ The motivation behind these studies is to draw lessons from nature that would enable the development of design rules applicable to manmade composites such that orders of magnitude improvements in performance over their constituents can be achieved.

Tablets of nacre have been investigated in the past, but their exact structure has not been fully elucidated. Transmission electron microscopy from Sarikaya et al. ${ }^{1}$ showed that each of the tablets is composed of 4-6 twinned aragonite grains. A strong texture perpendicular to the surface of the tablets was observed. ${ }^{1,3,4}$ On the other hand, from atomic force microscopy observations, $\mathrm{Li}$ et al. ${ }^{5}$ recently suggested that the tablets are actually composed of nanograins. In addition, the biomineralization of the tablets leaves some soluble proteins within the tablets, as intracrystalline inclusions. ${ }^{6}$

Understanding what makes nacre so tough also requires some quantitative data on its mechanical properties. The mechanical behavior of nacre has been studied experimentally using a variety of configurations ${ }^{7-10}$ and strain rates. ${ }^{11}$ However, almost no attempts have been made to directly probe the mechanical properties of the individual components of nacre. Katti et al. ${ }^{12}$ performed indentation on individual tablets, but through the use of indentation and standard data reduction techniques, ${ }^{13}$ the effect of roughness and underlying interfaces was neglected. In addition, the mechanical properties of the interfaces were estimated by indenting material on the surface of a cleaved nacre specimen, which may have been 

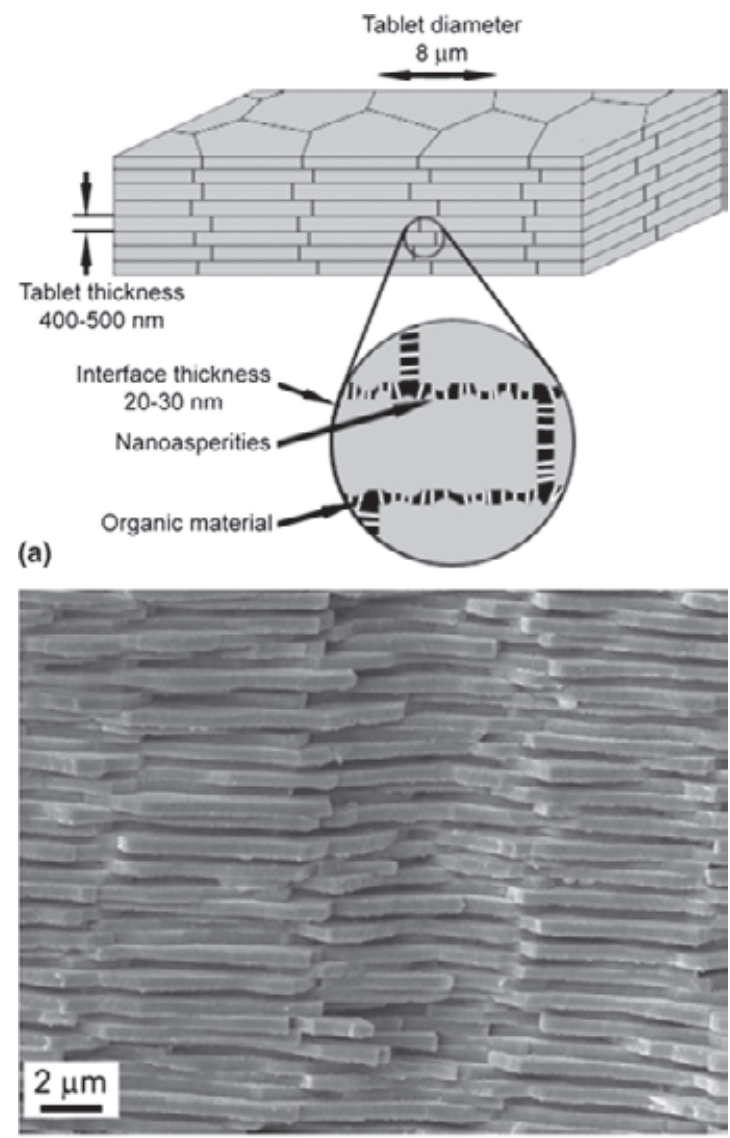

(b)

FIG. 1. (a) Schematic illustration of some of the features of nacre microstructure. (b) Scanning electron micrograph of a fracture surface of red abalone nacre showing the tablet stacking arrangement.

damaged during the cleavage of the sample and consequently might not reflect the properties of intact interfaces. In a simultaneous study, Bruet et al. ${ }^{14}$ performed nanoindentation experiments on cleaved nacre from Trochus Niloticus (top shell). The effects of the softer interfaces were neglected to determine the modulus and hardness of the tablets. The present work on nacre from Haliotis Rufescens (red abalone) actually shows that the underlying surfaces affect the indentation results. Smith et al. ${ }^{15}$ used an atomic force microscope (AFM) cantilever tip to stretch single biomolecules from a cleaved nacre surface. This experiment showed the significant deformation capability of the biopolymer, but no connection to the bulk properties of the interface was made. Because of the lack of accurate material parameters, various moduli have been used in the past for modeling purposes; for the tablets, Young's moduli of $100,{ }^{8} 70,{ }^{16}$ and $50 \mathrm{GPa}^{17}$ were used, and for the interface $20 \mathrm{MPa}^{12}$ was used. While approximate values are acceptable for outlining trends and mechanisms, more accurate material properties are needed to model all the synergistic effects involved in the deformation of nacre. In this work, the structure of tablets and interfaces are discussed based on transmission electron microscope (TEM), scanning electron microscope (SEM), and AFM observations. A method for measuring the moduli and strengths of both tablets and interfaces was recently presented. ${ }^{18}$ The method is elaborated here, and the results are compared with existing data for single-crystal aragonite. The experimental findings on the morphology of nacre, in particular those concerning the dimensions and density of the nanoasperities extracted from our AFM and TEM imaging and the identified material parameters, are then used to model one of the deformation mechanisms occurring in nacre when it is loaded in the in-plane direction.

\section{STRUCTURE OF TABLETS AND INTERFACES}

Nacre specimens were harvested from the nacreous layer of a red abalone specimen (Haliotis rufescens). TEM samples of nacre were prepared using standard grinding and ion milling techniques. "Face-on" specimens were prepared for viewing along the normal to the tablets. "Edge-on" specimens were prepared for viewing across the tablets. TEM and selected area diffraction (SAD) images of the tablets face-on [along the normal to the tablets, Fig. 2(a)] and edge-on [along the plane of the tablets, Fig. 2(b)] were obtained. The spot diffraction pattern was indexed as aragonite, with orientation consistent with the [001] direction normal to the tablets. Nanograins embedded in the crystal were also observed in both views, which translated into rings on the SAD patterns. Figure 3 shows a high-resolution TEM of the nanograins. We did not find all tablets made only of nanograins as argued by $\mathrm{Li}$ et al. ${ }^{5}$ The interfaces, namely the $20-40 \mathrm{~nm}$ thin layers between the aragonite tablets, are complex systems composed of several species of biomolecules organized in several distinct layers. ${ }^{19}$ Across these organic layers, nano-sized aragonite asperities on the surface of the tablets are believed to play a significant role in the toughening of nacre. ${ }^{16}$ Aragonite bridges across the interfaces are also believed to provide direct mechanical connections between tablets. ${ }^{20}$ TEM imaging (Fig. 4) reveals that both direct connections and asperities are present at the interface.

\section{NANOINDENTATION EXPERIMENTS}

To probe the mechanical properties of both tablets and interfaces, nanoindentation tests were performed on nacre. Nanoindentation is the method of choice to interrogate small volumes of material, in this case a single nacre tablet. However, it is an indirect characterization technique that requires special data reduction procedures in the case of multilayered materials such as nacre. In this work, the indentation specimens were small cubes (about $2 \times 2 \times 2 \mathrm{~mm}$ in size) harvested from the nacreous layer 


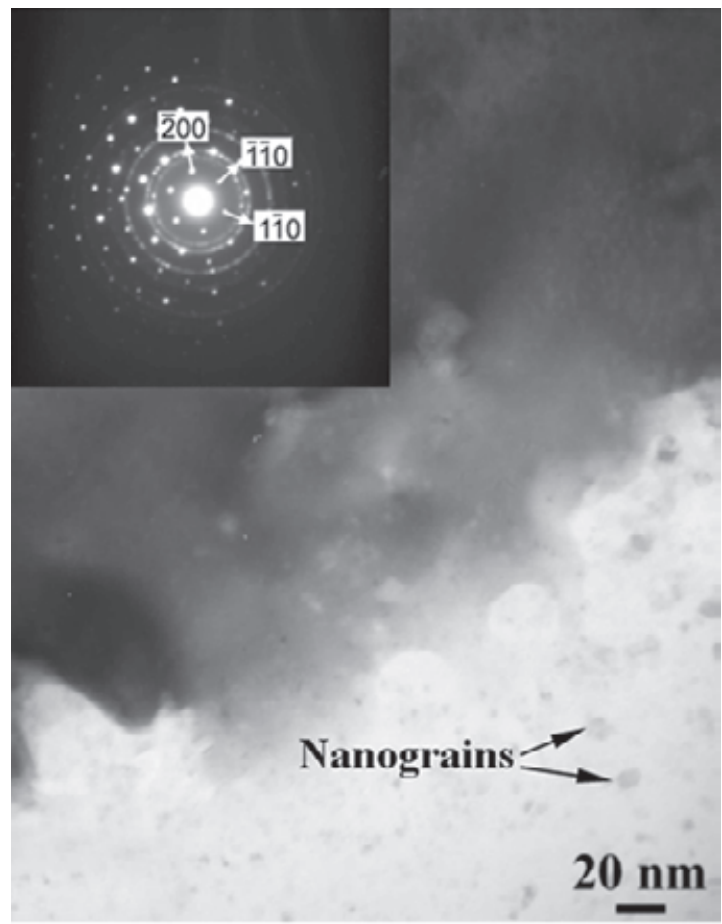

(a)

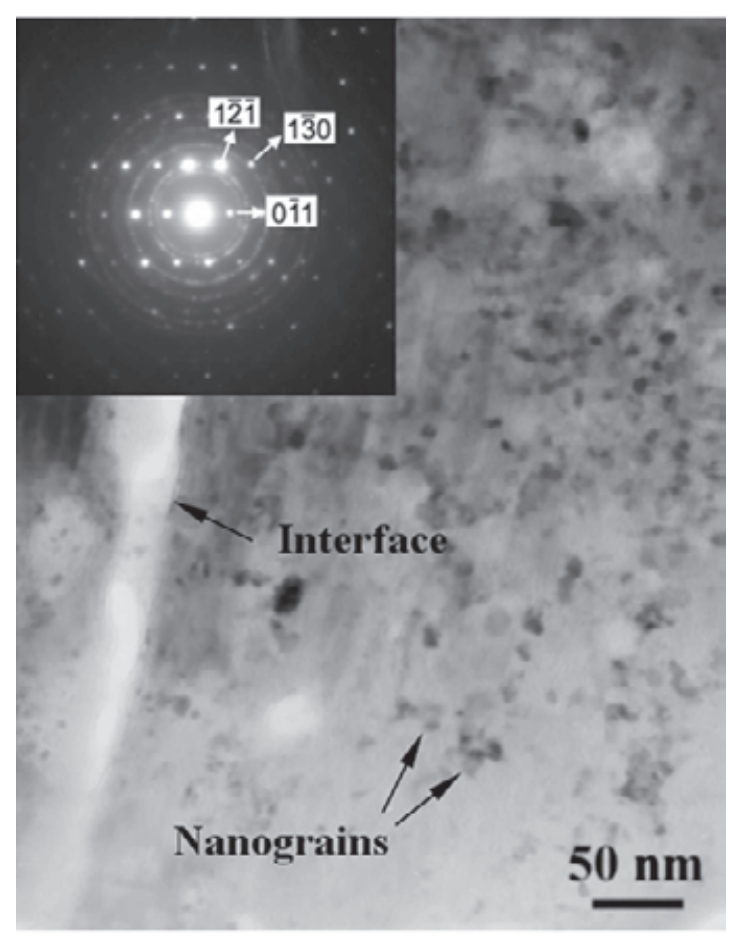

(b)

FIG. 2. TEM microstructure of the nacre: (a) face-on view within a tablet, $\mathrm{B} / /[001]$ and (b) edge-on view, $\mathrm{B} / /[311]$.

of a red abalone specimen. Using a razor blade, the specimens were cleaved along the direction of the tablets. The organic layer covering the cleaved faces was removed by plasma etching, and the surfaces were investigated by AFM. Characterization of the surface revealed nanoscale

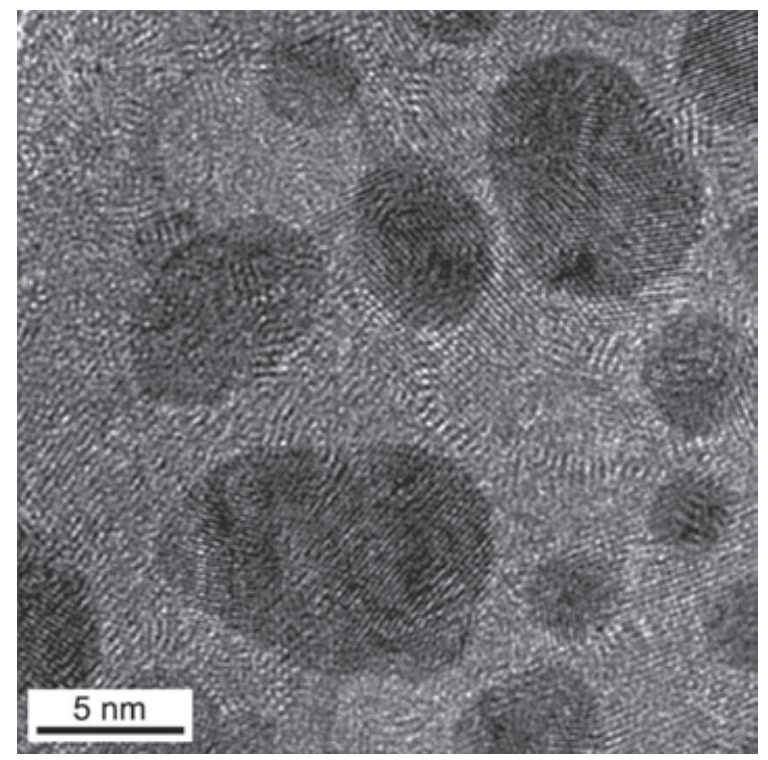

FIG. 3. High-resolution TEM (face-on view) of a tablet showing nanograins about $3-10 \mathrm{~nm}$ in size.

asperities covering the surface of the tablets (Fig. 5) as described previously. ${ }^{10}$ On average, the height of these asperities varied from 10 to $30 \mathrm{~nm}$ while their spacing was on the order of 100-200 nm. The density, size, and shape of these asperities can vary significantly from one area to another.

The nacre specimens were glued onto a sample holder, and nanoindentation tests were performed on the cleaved surface using a diamond Berkovich tip with a Nanoindenter XP system (MTS Systems Corporation, Eden Prairie, MN). Nacre was in the dried (ambient) condition for these experiments. The indentations were performed under load-controlled conditions at a load rate of $2 \mu \mathrm{N} / \mathrm{s}$. Load-controlled indentation was preferred over displacement-controlled indentation because the variation of roughness on the surface makes it difficult to determine the actual indentation depth. Toward the end of the unloading stage, the load was held for $30 \mathrm{~s}$ to perform thermal drift corrections. ${ }^{13}$ Figure 6 shows typical load-deflection curves ( $P$ - $\delta$ curves) and indents left on the surface. The deeper indentation tests were performed at maximum indentation loads of about $1.6 \mathrm{mN}$. The resulting curves [Fig. 6(a)] showed a peculiar feature: sudden displacement jumps occurred at regular intervals on the $P-\delta$ curve (these events were captured only when a high data acquisition rate was used). This phenomenon can be attributed to the collapse and densification of the interface between the tablets. The interfaces collapsed in a quasi-periodic fashion, starting from the first interface and spreading to deeper interfaces as the indentation depth increased.

The indents left on the surface after the experiments were imaged using AFM [Fig. 6(b)]. No radial cracking emanating from the corners of the indents could be 


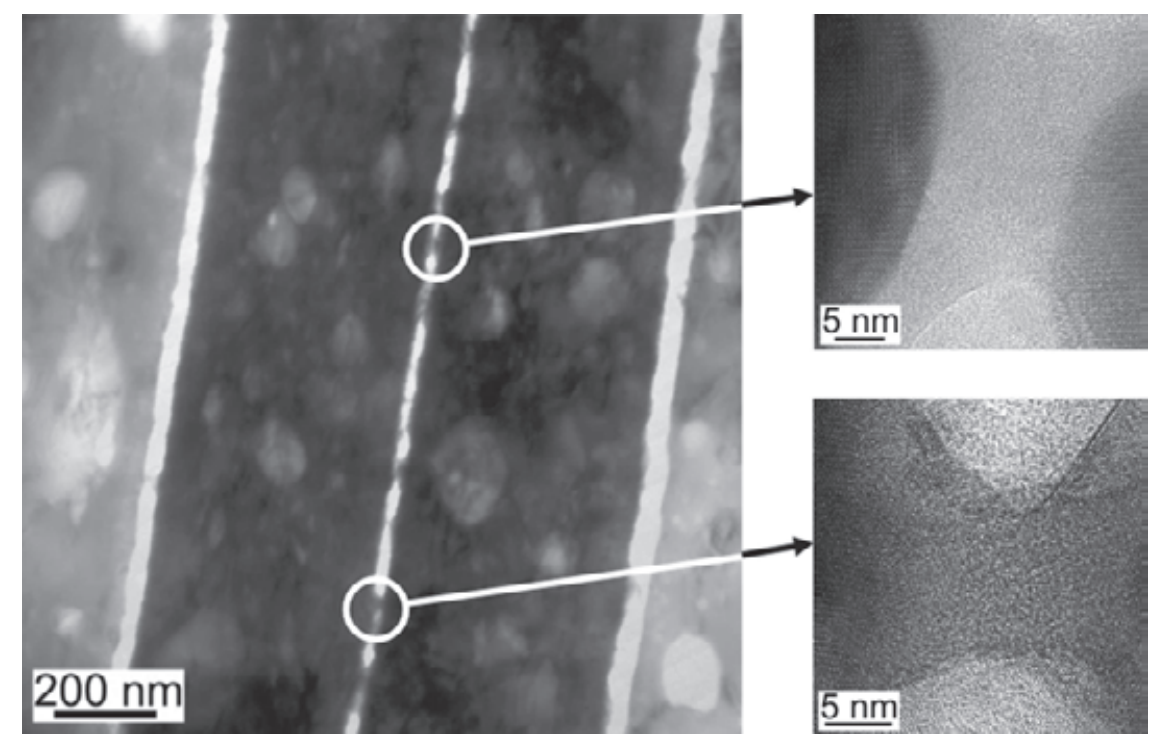

FIG. 4. TEM micrograph showing the thin interfaces between the tablets. Both asperities (upper) and direct connections (lower) were found across the interfaces.

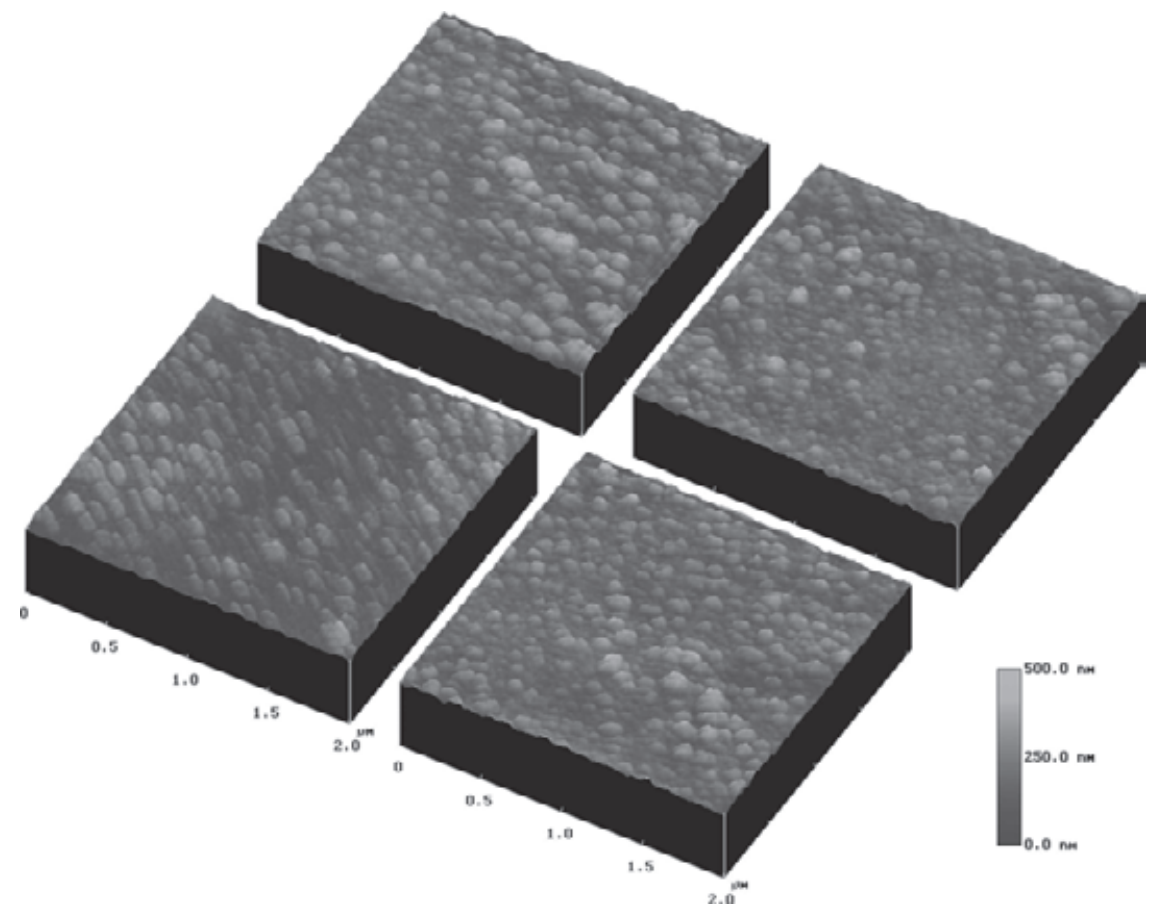

FIG. $5.2 \times 2 \mu \mathrm{m}$ AFM scans from different areas of a cleaved specimen of nacre, showing the surface of the tablets. Asperities of various densities, heights, and shapes could be observed.

observed. The absence of cracks is not surprising at such low depth indentation regime, i.e., when the indentation load is lower than the critical value for crack initiation. ${ }^{21}$ For very low indentation depth experiments, the maximum load used was $0.1 \mathrm{mN}$, and the indenter was unloaded before the first collapse occurred. The curves show an initial region with a small slope, which is attributed to the plastic flattening of the nanoasperities. The length of this region varies from one indent to the next because of the variation in local roughness. After a displacement of $20-30 \mathrm{~nm}$ (which corresponds to the height of the asperities), the indenter engaged the bulk of the tablets, and the stiffness increased rapidly. There is limited plastic deformation in the bulk of the material, and AFM showed no obvious indent. The indent can be located as it leaves a round area on the surface where the asperities were flattened. The $P-\delta$ curves from these experiments were first processed using the method 

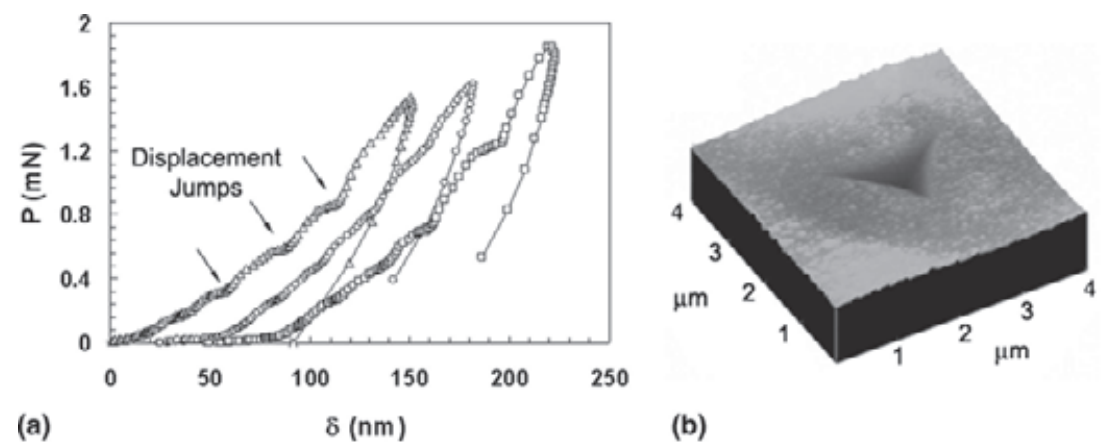

(a)

(b)
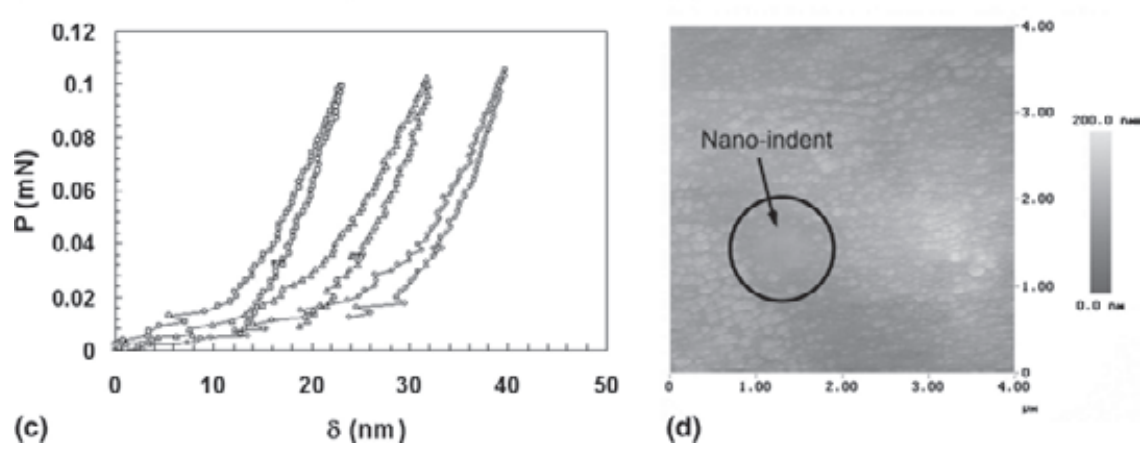

(d)

FIG. 6. (a) Large depth indentation. The curves were shifted horizontally for clarity. Note the softening events during the loading stage. (b) AFM image of the indent. The indent showed neither pile-up nor cracking. (c) Low depth indentation. These curves were not shifted horizontally, but variation in local roughness led to significant horizontal scattering. (d) AFM image of the indent. The location of the indent corresponds to local asperity flattening.

developed by Oliver and Pharr. ${ }^{13}$ The results are shown in Fig. 7 in terms of the reduced modulus, which is written as function of the elastic constants of the tablets and indenter:

$$
\frac{1}{E_{\mathrm{R}}}=\frac{\left(1-v_{\mathrm{t}}^{2}\right)}{E_{\mathrm{t}}}+\frac{\left(1-v_{\mathrm{ind}}{ }^{2}\right)}{E_{\text {ind }}},
$$

and of the hardness. The data present significant scatter in the low depth regime (due to surface roughness effect) that decreases as the depth increases. The results also show that both apparent modulus and hardness decrease when the indentation depth increases. This trend could be related to two phenomena: the first is the dependence of strength on strain gradient at the nano and microscale. ${ }^{22}$ The second, believed to be predominant in this case, is the presence of the interfaces; the softer interfaces can be "felt" by the indenter and have a significant impact on the results. This type of effect was also reported for indentation of a thin film on a softer substrate: substrate properties influence the results already for indentation depths less than half the film thickness (see Saha et al. ${ }^{23}$ or Bhushan et al. ${ }^{24}$ for example).

\section{PARAMETER IDENTIFICATION}

From these results, it is clear that the effect of the softer interface needs to be included in the analysis. To capture the contribution of the interface, the experiment was modeled using finite elements, and the material properties were found by fitting the experimental curve with the model. Figure 8 shows the finite element model of the indenter and the nacre specimen generated using ANSYS (ANSYS Inc., Canonsburg, PA). The exact topology of the Berkovich indenter tip used in the experiments was determined using AFM. Rounding of the tip of the indenter was identified and quantified. We noted that in the low indentation depth experiments, the indenter could be modeled as axisymmetric. The exact shape of the indenter was determined from the actual shape area function obtained from the AFM scan. The diamond indenter was modeled as elastic with properties $E=1140 \mathrm{GPa}$ and $v=0.07$. The substrate was modeled as an axisymmetric layered structure with 450-nmthick tablets and 35-nm-thick interfaces (these values are based on actual measurement from micrographs). Parametric studies on the size of the model showed that three tablets and two interfaces with a radius of $1500 \mathrm{~nm}$ were sufficient to model a half-space of nacre subjected to low depth indentation $(\delta<20 \mathrm{~nm})$.

The tablets were modeled as isotropic elastic-perfectly plastic; their behavior is thus defined by three material parameters: Young's modulus $E_{\mathrm{t}}$, Poisson's ratio $v_{\mathrm{t}}$, and the compressive strength $\sigma_{\mathrm{t}}$. The interfaces are complicated systems containing biopolymers, and a rigorous treatment of their mechanical behavior would probably require time-dependent models. In this work, however, 

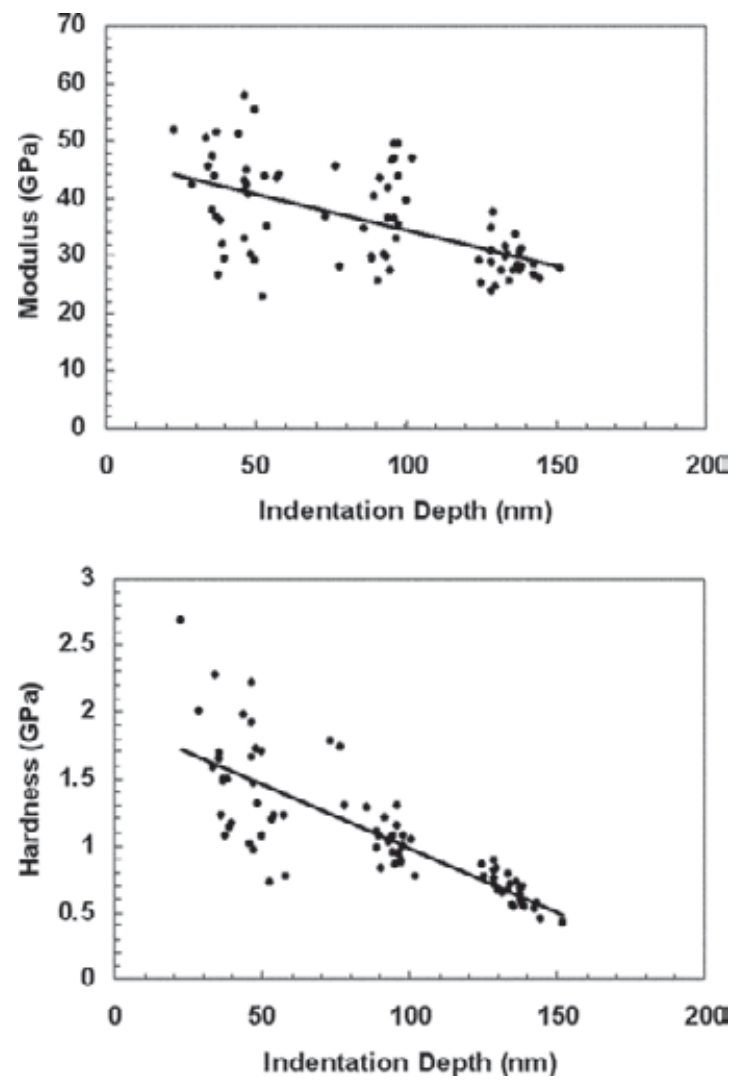

FIG. 7. Modulus and hardness as predicted using standard analysis technique.

the interfaces were modeled as linear elastic (with elastic parameters $E_{\mathrm{i}}$ and $v_{\mathrm{i}}$ ) for the sake of simplicity and to limit the numbers of material parameters. These five parameters can in principle be identified by inverse analysis by fitting the experimental and numerical $P-\delta$ curves. However, this inverse problem is ill-posed, and the identification can lead to different sets of material parameters. In particular, as is well known in literature, the sensitivity of indentation results to Poisson's ratio is very low, and their values can hardly be identified by inverse analysis. Because of the non-uniqueness of the solution, additional experimental data are required to add constraints to the identification procedure.

To provide additional experimental data, other independent experimental tests were performed, namely macroscopic uniaxial compression tests perpendicular to the tablets. The specimens used were small prisms, about 2 $\mathrm{mm}$ long with a cross section of about $1 \times 1 \mathrm{~mm}$. As in the indentation test, the specimens were tested in dry conditions. The specimen was placed between two alumina blocks inside a miniature loading stage (Ernest F. Fullam, Inc., Latham, NY). The setup was then placed under an optical microscope, and pictures of the specimen were taken at regular interval during the loading. The pictures were used to determine displacements and strains by means of a digital image correlation algorithm
(VIC2D, Correlated Solutions, West Columbia, SC). Two experiments were performed, with good reproducibility (Fig. 9). The stress-strain curves showed a linear behavior with slope $E_{\mathrm{c}}=29 \mathrm{GPa}$. Note that this value is consistent with the modulus from indentation for the largest depths. The specimens failed at compressive stresses ranging from 400 to $600 \mathrm{MPa}$. The technique also yielded the transverse strains, from which a Poisson's ratio of $v_{\mathrm{c}}=0.16$ was measured.

The elastic behavior of nacre when loaded across the tablets can be modeled as a Reuss composite, where stress is equal in both tablets and interfaces. Typically, the Reuss model involves the Young's moduli of both phases. In this case, however, the interfaces are much softer and thinner than the tablets. They are confined by the tablets, and they experience a multiaxial state of stress. By combining the equations of elasticity in tablets and interfaces, it was shown that the composite modulus $E_{\mathrm{c}}$ is given by:

$\frac{t_{\mathrm{t}}+t_{\mathrm{i}}}{E_{\mathrm{c}}}=\frac{t_{\mathrm{t}}}{E_{\mathrm{t}}}+\frac{t_{\mathrm{i}}}{E_{\mathrm{i}}^{*}}$ for $\frac{t_{\mathrm{i}}}{t_{\mathrm{t}}} \ll 1$ and $\frac{E_{\mathrm{i}}}{E_{\mathrm{t}}} \ll 1$,

where $t_{\mathrm{t}}, t_{\mathrm{i}}, E_{\mathrm{t}}$, and $E_{\mathrm{i}}$ are the tablets and interfaces thicknesses and moduli, respectively. The "confined" modulus $E_{\mathrm{i}}^{*}$ can be written as:

$$
E_{\mathrm{i}}^{*}=\frac{\left(1-v_{\mathrm{i}}\right)}{\left(1+v_{\mathrm{i}}\right)\left(1-2 v_{\mathrm{i}}\right)} E_{\mathrm{i}}
$$

where $E_{\mathrm{i}}$ and $\nu_{\mathrm{i}}$ are the modulus and Poisson's ratio for the interfaces. Note that $E_{\mathrm{i}}$ and $\nu_{\mathrm{i}}$ cannot be determined uniquely from the present experiments. A twodimensional finite element model based on the real microstructure of nacre (not shown here) predicted a modulus very close to the one given by the Reuss model. Therefore Eqs. (2) and (3) provide a simple way to predict the modulus of nacre across the tablets as function of tablet and interface moduli. As for the composite Poisson's ratio, a reasonable estimate is given by the rule of mixtures:

$$
\left(t_{\mathrm{t}}+t_{\mathrm{i}}\right) v_{\mathrm{c}}=t_{\mathrm{t}} v_{\mathrm{t}}+t_{\mathrm{i}} v_{\mathrm{i}}
$$

Because the tablets are much thicker than the interfaces one can assume that Poisson's ratio measured on nacre is equal to Poisson's ratio of the tablets:

$$
v_{\mathrm{t}} \approx v_{\mathrm{c}}=0.16 \text {. }
$$

The behavior of the interface is controlled by the confined modulus $E_{\mathrm{i}}{ }^{*}$, and the finite element code used for parameter identification requires a modulus and Poisson's ration input for the interfaces. One way to proceed is to set $v_{\mathrm{i}}=0.4$ for the interfaces (a typical value for polymers) and set $E_{\mathrm{i}}$ as a free fitting parameter. Upon convergence of the fitting procedure, the confined modulus $E_{\mathrm{i}}^{*}$ is then determined using Eq. (3). This means that 

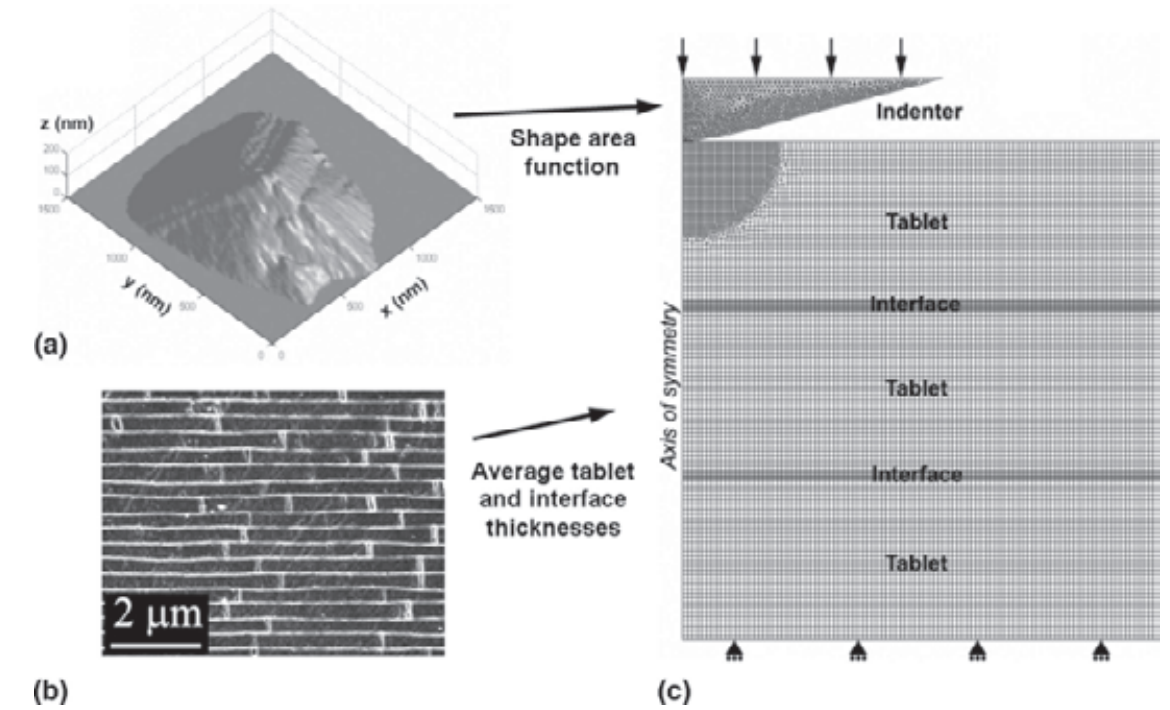

(c)

FIG. 8. (a) AFM image of the indenter, (b) SEM image showing the microstructure of nacre, and (c) Finite element model based on the actual shape of the Berkovich tip and the actual structure of nacre.

multiple pairs of $\left(E_{\mathrm{i}}, v_{\mathrm{i}}\right)$ could have led to the same $E_{\mathrm{i}}$, i.e., the same $P-\delta$ curve. For example, setting $\nu_{\mathrm{i}}=0$ would have yielded a different value for $E_{\mathrm{i}}$ but the same confined modulus $E_{\mathrm{i}}{ }^{*}$.

An automated fitting procedure (steepest descent method) was implemented to fit the model onto individual $P-\delta$ curves. By the use of the compression tests results and of the above assumptions, only two independent parameters were left to be identified. As discussed above, the roughness makes it difficult to determine the exact experimental indentation depth, and for this reason, the experimental curves were shifted horizontally by an amount added as a fitting parameter. In addition, a load cutoff of $0.06 \mathrm{mN}$ was used on the experimental curve to exclude the initial stages of the $P-\delta$ curves from the fitting procedure. The experimental and modeled curves

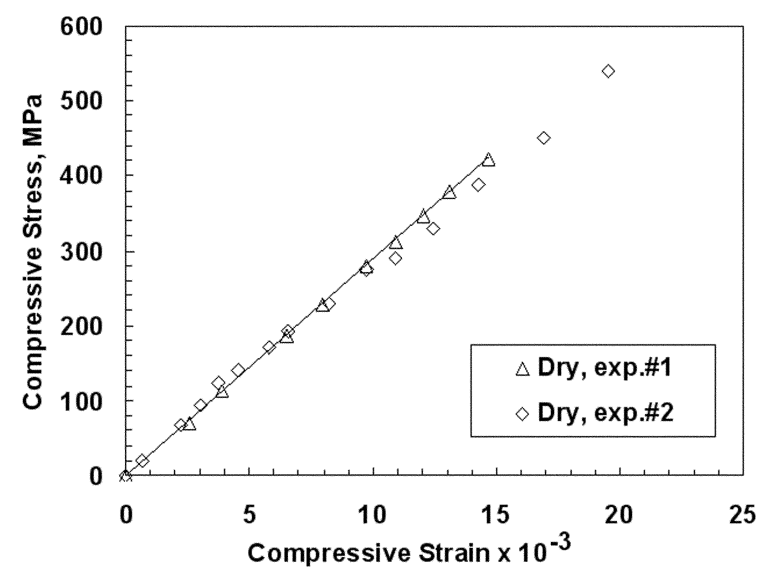

FIG. 9. Compressive stress-strain curves of nacre across the tablets. Dry specimens were used for consistency with the indentation experiments. were compared only for loads greater than this cutoff value. Figure 10 shows a typical best fit obtained using this method. The procedure was repeated over about $50 P$ - $\delta$ experimental curves, and statistics on the results yielded the following values for the tablets (the results are given as mean \pm standard deviation) $E_{\mathrm{t}}=79 \pm$ $15 \mathrm{GPa}, \sigma_{\mathrm{t}}=2.5 \pm 1.2 \mathrm{GPa}$, and for the interfaces $E_{\mathrm{i}}^{*}=2.84 \pm 0.27 \mathrm{GPa}$.

The fitting procedure converged to these values independent of the initial values for the optimization, which shows the effectiveness of the additional constraint [Eq. (2)] coming from the uniaxial compression results.

\section{DISCUSSION}

The measured elastic properties of the tablets can be compared with existing data. Single-crystal aragonite has

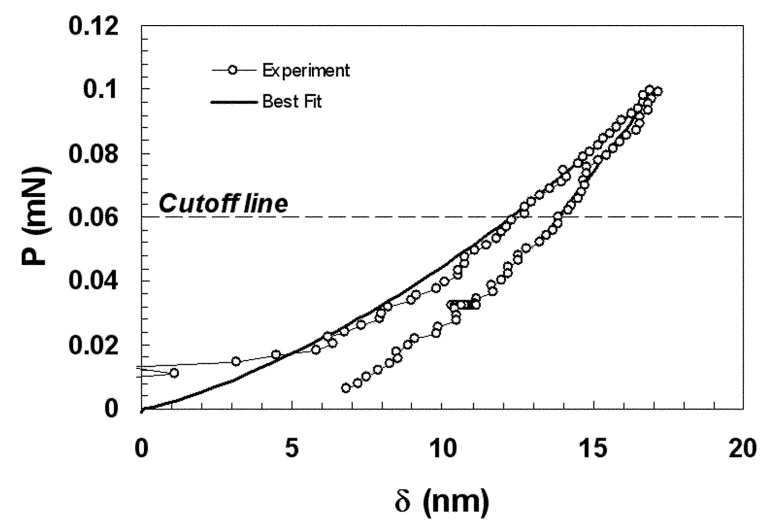

FIG. 10. Example of experimental and best-fit $P-\delta$ curves. Note that only the initial unloading slope is plotted for the model. 
orthotropic elastic properties, and its stiffness coefficients are well established ${ }^{25,26}$ :

$$
\begin{array}{lll}
E_{1}=144 \mathrm{GPa} & v_{12}=0.44 & G_{12}=47.2 \mathrm{GPa} \\
E_{2}=76 \mathrm{GPa} & v_{13}=-0.06 & G_{13}=25.6 \mathrm{GPa} \\
E_{3}=82 \mathrm{GPa} & v_{23}=0.18 & G_{23}=41.3 \mathrm{GPa}
\end{array}
$$

A finite element model of a rigid sphere indenting a single-crystal aragonite substrate was used to determine the reduced modulus of single-crystal aragonite along the [001], which was found to be $82.7 \mathrm{GPa}$. This compares very well with the reduced modulus found here $(81 \mathrm{GPa})$, which shows that despite the existence of nanograins and organic molecules (inclusions), the elastic properties of the tablets are basically those of single-crystal aragonite. The compressive strength of the tablets was also determined. Previous work by Han et al. ${ }^{27}$ presented Knoop hardness results on single-crystal aragonite, and strong indentation size effect was demonstrated for this material. The hardness found in that work ranged from $3 \mathrm{GPa}$ for $2 \mathrm{~N}$ indentation load to $4.5 \mathrm{GPa}$ for $0.25 \mathrm{~N}$ indentation load along the [001] crystallographic direction. Approximating the compressive strength as $1 / 3$ of the hardness, the corresponding strength would range from 1 to $1.5 \mathrm{GPa}$ for the lowest load $(250 \mathrm{mN})$. In this work, a strength of $2.5 \mathrm{GPa}$ was found using a much smaller load $(0.1 \mathrm{mN})$. This higher value is consistent with plasticity scale effects but can also be explained by the presence of nanograins within the tablets, which act as dislocation barriers. The yield strength of aragonite found here can also be correlated to the flattening stage of the indentation experiment. Figure 6(c) shows that the asperities are completely flattened at a load of about $0.02 \mathrm{mN}$. During the flattening stage, only a limited amount of material is in contact with the indenter. By using the flow stress of $2.5 \mathrm{GPa}$ found above, one can estimate the actual indentation contact area by $A=0.02 \mathrm{mN} / 2.5 \mathrm{GPa}=$ $8000 \mathrm{~nm}^{2}$. Assuming axisymmetric asperities of diameter equal to $40 \mathrm{~nm}$, the cross-sectional area of a single asperity is about $1200 \mathrm{~nm}^{2}$. From these calculations, the number of flattened asperities can be computed: $8000 / 1200=$ about 6 to 7 asperities. This estimate is consistent with the AFM scan of Fig. 6(d): the imprint left by the indent can easily contain 6-7 nanoasperities. The modulus of the interface $\left(E_{\mathrm{i}}^{*}=2.84 \pm 0.27 \mathrm{GPa}\right)$ is, as expected, much lower than the stiffness of the tablets. This value is, however, much higher than the one reported by Katti et al. $\left(20 \mathrm{MPa}^{12}\right)$. This discrepancy can probably be explained by the fact that Katti et al. measured the stiffness of interface material covering cleaved surfaces, which probably included only the organic material. In the present case, the modulus was determined from intact interfaces, and it includes additional stiffening factors such as asperity-asperity contact and load transfer through aragonite bridges.

\section{MODEL FOR ASPERITIES INTERACTIONS}

Sliding of the tablets is believed to be one of the main toughening mechanisms in nacre because it allows for significant macroscopic deformations and some ductilelike behavior. It has been suggested that the nanoasperities on the surface of the tablets provide resistance to tablet sliding and local hardening necessary to the spreading of deformation throughout the material. ${ }^{10,16}$ Given the high stresses involved in contact mechanisms, plastic deformations of the asperities might have a strong influence on the overall response. To investigate this aspect, the asperity model proposed by Evans et al. ${ }^{16}$ was examined using ANSYS. The dimensions of the asperities used in the model here presented are different, however, and they follow more closely the statistics extracted from our AFM and TEM imaging. The model consists of two-dimensional periodic representative elements that include a $1-\mu \mathrm{m}$ sliding region covered with asperities. Individual asperities were modeled with a sine wave profile, exhibiting a height of $20 \mathrm{~nm}$ and a width of $40 \mathrm{~nm}$. The spacing between asperities is $100 \mathrm{~nm}$, and the interface thickness was set to $35 \mathrm{~nm}$. As a result, the asperityasperity inception was $5 \mathrm{~nm}$ (Fig. 11). Contact elements were inserted on the opposing faces, and the local friction was set to zero. First, the case of a linear elastic material was considered. Asperity climb provides resistance to tablet sliding at the nanoscale, which in turn generated a macroscopic tensile stress. Figure 11(d) shows the resulting tensile stress-strain curve predicted by the model. The maximum tensile stress generated in the linear elastic case is $215 \mathrm{MPa}$, which is greater than the experimental value $(85 \mathrm{MPa})$. In a second model, perfect plasticity was added with the strength of aragonite set to $2.5 \mathrm{GPa}$. For this case, the predicted tensile strength is $190 \mathrm{MPa}$, which is slightly lower than the linear elastic case. Asperity climb induces very little plasticity in the asperities. This demonstrates that the aragonite asperities are strong enough to generate significant resistance to tablet sliding. However, the predicted tensile strengths are significantly higher than the experimental values. Note that the architecture of the interface is highly idealized in this model. In reality, some of the asperities may not contact each other (Fig. 4) so the resistance provided by climbing is probably lower. An important feature of the model is that instability occurs at a strain of about $0.45 \%$ (the model could not numerically converge beyond this point). The experimental tensile stress-strain curve shows strain hardening up to failure, which occurs at about $1 \%$ strain. A similar observation can be made in the nanoasperity model in Ref. 16 . The nanoasperities can only provide shear resistance over sliding distances of only $20-25 \mathrm{~nm}$, which is much smaller than the sliding distances observed experimentally $(100-200 \mathrm{~nm}){ }^{10}$ Therefore, the nanoasperities model does not appear to 


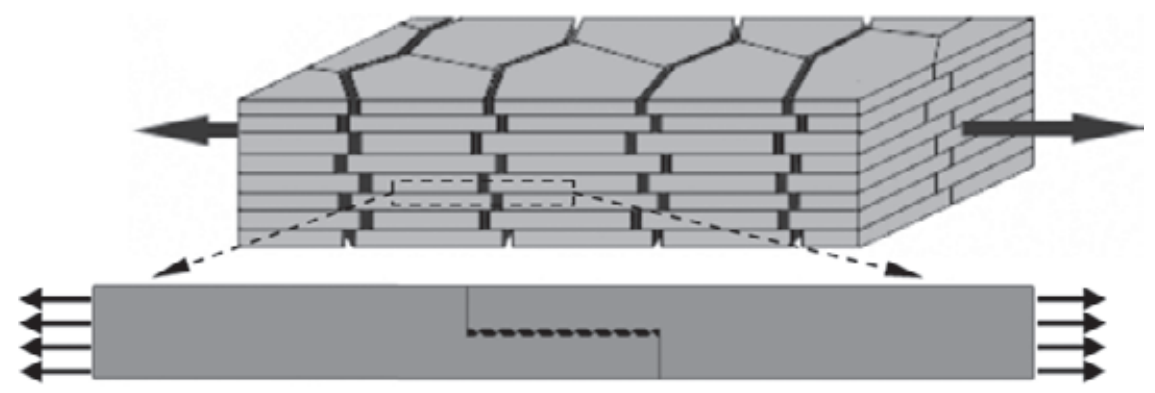

(a)

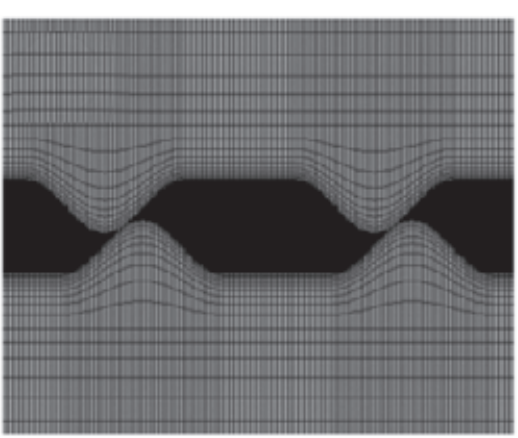

(b)

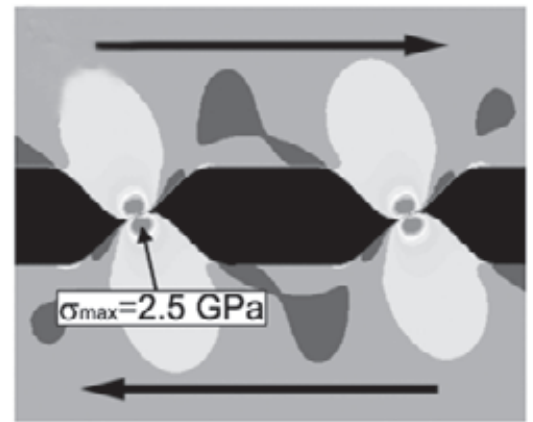

(c)

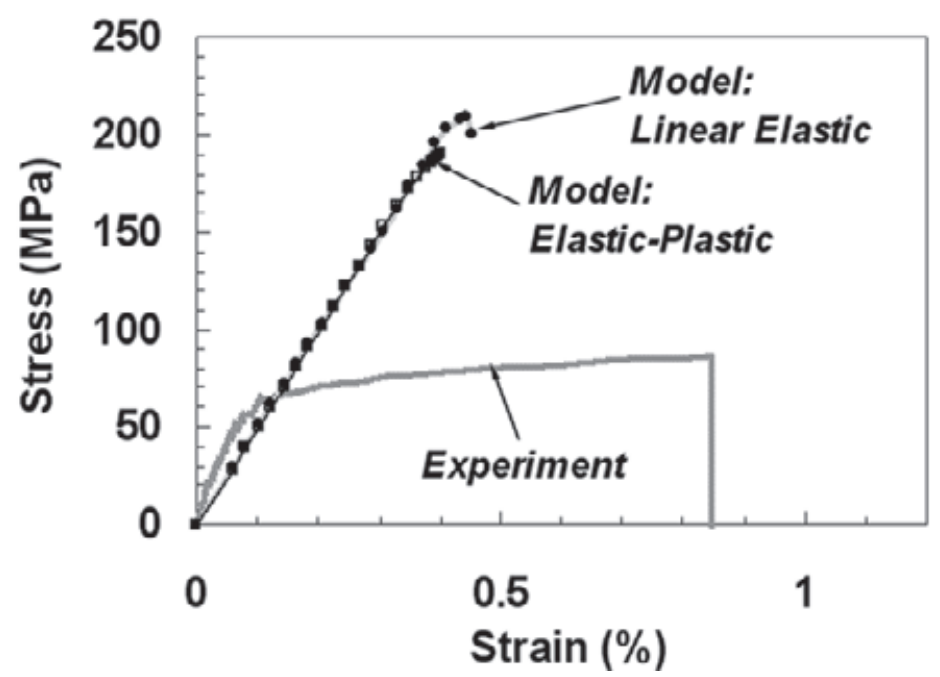

FIG. 11. (a) Overview of the model, (b) detail of the mesh with the two sides in contact, and (c) Von Mises stresses generated by contact between asperities. (d) Experimental tensile stress-strain curve and as predicted by the finite element models using linear elastic and elastic-plastic models.

capture the sliding resistance and hardening measured experimentally. This suggests that other microstructural features must be responsible for the observed cohesive strength between the tablets over larger sliding distances.

\section{CONCLUSIONS}

In summary, the structure of the tablets and their mechanical properties was investigated on nacre from Haliotis rufescens (red abalone). The tablets were primarily composed of large aragonite grains with some inclusions of nanograins. The softer interfaces were shown to have a significant effect on nanoindentation results. A finite element model that included the interface was therefore used to determine relevant single tablet mechanical properties. The elasticity of the tablets was found to be close to that of single aragonite crystals. Nanograins and organic material inclusion do not seem to significantly affect the elasticity of the tablets. The indentations did not generate any cracking in the tablets, but this does not imply that the material is not brittle. It is known that below a critical indentation load, all materials, including glass, sapphire, alumina, etc., will behave plastically with no cracking. Given the crystallography of the tablets, it is actually likely that the tablets are brittle in tension. They can, however, rely on their small 
size for improved strength because the critical flaw size for aragonite is larger than that of the tablets. ${ }^{7,28}$ The compressive strength of the tablets was found to be higher than existing indentation data on single-crystal aragonite. This can be attributed to size effects (our data was obtained from lower indentation depths) and/or to the presence of the nanograins, which may act as obstacles to dislocations. An investigation of the model proposed by Evans et al., ${ }^{14}$ incorporating the tablet properties identified in this work, suggests that the nanoasperities and nanograins are not likely to be the key morphological features controlling the unique mechanical response of nacre. We must therefore investigate further to identify the most relevant features of nacre that, when combined with the properties of its constituents, lead to superior material properties.

\section{ACKNOWLEDGMENTS}

This work was supported by the National Science Foundation through award No. CMS-0301416. We would like to thank Dr. Ken Chong for his encouragement and support during this investigation. C. Comi is grateful for the kind hospitality and the financial support of the Department of Mechanical Engineering at Northwestern University.

\section{REFERENCES}

1. M. Sarikaya and I.A. Aksay: Biomimetics, design and processing of materials, in Polymers and Complex Materials, edited by M. Sarikaya and I. Aksay, (AIP, Woodbury, NY, 1995).

2. G. Mayer: Rigid biological systems as models for synthetic composites. Science 310, 1144 (2005).

3. Q.L. Feng, F.Z. Cui, G. Pu, R.Z. Wang, and H.D. Li: Crystal orientation, toughening mechanisms and a mimic of nacre. Mater. Sci. Eng., C-Biomimetic Supramolecular Syst. 11, 19 (2000).

4. E. DiMasi and M. Sarikaya: Synchrotron x-ray microbeam diffraction from abalone shell. J. Mater. Res. 19, 1471 (2004).

5. X.D. Li, W.C. Chang, Y.J. Chao, R.Z. Wang, and M. Chang: Nanoscale structural and mechanical characterization of a natural nanocomposite material: The shell of red abalone. Nano Lett. $\mathbf{4}$ $613(2004)$.

6. A.M. Belcher, X.H. Wu, R.J. Christensen, P.K. Hansma, G.D. Stucky, and D.E. Morse: Control of crystal phase switching and orientation by soluble mollusc-shell proteins. Nature $\mathbf{3 8 1}$. $56(1996)$.

7. J.D. Currey: Mechanical properties of mother of pearl in tension. Proc. R. Soc. London 196, 443 (1977).

8. A.P. Jackson, J.F.V. Vincent, and R.M. Turner: The mechanical design of nacre. Proc. R. Soc. London 234, 415 (1988).

9. R.Z. Wang, H.B. Wen, F.Z. Cui, H.B. Zhang, and H.D. Li: Observations of damage morphologies in nacre during deformation and fracture. J. Mater. Sci. 30, 2299 (1995).
10. R.Z. Wang, Z. Suo, A.G. Evans, N. Yao, and I.A. Aksay: Deformation mechanisms in nacre. J. Mater. Res. 16, 2485 (2001).

11. R. Menig, M.H. Meyers, M.A. Meyers, and K.S. Vecchio: Quasistatic and dynamic mechanical response of haliotis rufescens (abalone) shells. Acta Mater. 48, 2383 (2000).

12. D.R. Katti, K.S. Katti, J.M. Sopp, and M. Sarikaya: 3D finite element modeling of mechanical response in nacre-based hybrid nanocomposites. Comp. Theor. Polvm. Sci. 11, 397 (2001).

13. W.C. Oliver and G.M. Pharr: An improved technique for determining hardness and elastic-modulus using load and displacement sensing indentation experiments. J. Mater. Res. 7, 1564 (1992).

14. J.F. Bruet, H.J. Qi, M.C. Boyce, R. Panas, K. Tai, L. Frick, and C. Ortiz: Nanoscale morphology and indentation of individual nacre tablets from the gastropod mollusc Trochus niloticus. J. Mater. Res. 20, 2400 (2005).

15. B.L. Smith, T.E. Schaeffer, M. Viani, J.B. Thompson, N.A. Frederick, J. Kindt, A. Belcher, G.D. Stucky, D.E. Morse, and P.K. Hansma: Molecular mechanistic origin of the toughness of natural adhesives, fibres and composites. Nature 399. 761 (1999).

16. A.G. Evans, Z. Suo, R.Z. Wang, I.A. Aksay, M.Y. He, and J.W. Hutchinson: A model for the robust mechanical behavior of nacre. J. Mater. Res. 16, 2475 (2001).

17. K. Okumura and P.G. de Gennes: Why is nacre strong? Elastic theory and fracture mechanics for biocomposites with stratified structures. Eur. Phvs. J. E. 4, 121 (2001).

18. F. Barthelat and H.D. Espinosa: Mechanical properties of nacre constituents: An inverse method approach, in Mechanical Properties of Bioinspired and Biological Materials, edited by C. Viney, K. Katti, F-J. Ulm, and C. Hellmich (Mater. Res. Soc. Symp. Proc. 844, Warrendale, PA, 2005), Y7.5.

19. T.E. Schaffer, C.I. Zanetti, R. Proksch, M. Fritz, D.A. Walters, N. Almqvist, C.M. Zaremba, A.M. Belcher, B.L. Smith, G.D. Stucky, D.E. Morse, and P.K. Hansma: Does abalone nacre form by heteroepitaxial nucleation or by growth through mineral bridges? Chem. Mater. 9, 1731 (1997).

20. F. Song, A.K. Soh, and Y.L. Bai: Structural and mechanical properties of the organic matrix layers of nacre. Biomaterials 24,3623 $\underline{(2003)}$.

21. B.R. Lawn: Fracture of Brittle Solids, 2nd ed. (Cambridge University Press, New York, 1993), pp. 282-295.

22. N.A. Fleck and J.W. Hutchinson: A phenomenological theory for strain gradient effects in plasticity. J. Mech. Phvs. Solids. 41, 1825 (1993).

23. R. Saha and W.D. Nix: Effects of the substrate on the determination of thin film mechanical properties by nanoindentation. Acta Mater. 50, 23 (2002).

24. B. Bhushan and X.D. Li: Nanomechanical characterisation of solid surfaces and thin films. Int. Mater. Rev. 48, 125 (2003).

25. F.S. Hearmon: The elastic constants of anisotropic materials. Rev. Mod. Phvs. 18, 409 (1946).

26. M. Levy, H. Bass, and R. Stern: Handbook of Elastic Properties of Solids, Liquids and Gases (Elsevier, San Diego, CA, 2001).

27. Y.H. Han, H. Li, T.Y. Wong, and R.C. Bradt: Knoop microhardness anisotropy of single-crystal aragonite. J. Am. Ceram. Soc. $\mathbf{7 4}$ 3129 (1991).

28. H.J. Gao, B.H. Ji, I.L. Jager, E. Arzt, and P. Fratzl: Materials become insensitive to flaws at nanoscale: Lessons from nature. Proc. Natl. Acad. Sci. USA 100, 5597 (2003). 